\title{
Evergreen Wheat: Using Genetic Modification to Generate a Perfect Blend of All Seasonal Varieties - A Review
}

\author{
${ }^{1}$ Kapoor Vidushi, ${ }^{2}$ Tandon Prateek \\ ${ }^{1,2}$ School of Bio-Sciences and Technology, Indian Institute of Technology Guwahati (IIT-G) and Carnegie \\ Mellon University, USA
}

\begin{abstract}
In recent years, with advent of the development of efficient plant regeneration systems in cereal crops, the field of recombinant DNA technology has opened up new avenues for genetic transformation of crop plants. This paper describes the genes involved in the wheat grown in different seasons namely winter and summer. The concerned genes have been studied and the paper makes an attempt towards a union of both varieties to produce a wheat that can be grown throughout the year (evergreen).The first winter group includes the regulatory regions of VRN1 and VRN3 genes or in the coding regions of VRN2. CBF transcription factors are known regulators of the COR genes (COld Regulated genes) which confer tolerance to subsequent freezing temperatures (acclimation). The WCS120 gene is specifically induced by low temperature (LT) and encodes a protein that is thought to play an important role in the cold acclimation process in wheat. The second summer group includes the basic two heat shock genes, hsp17 and hsp70. These genes regulate the heat shock condition of the wheat crop. Researchers have found out introducing a novel. DREB transcriptional factor into wheat is an effective way to improve its drought-tolerance ability. It has also been found that the $5 \mathrm{~A}$ chromosome plays a major regulatory role in $\mathrm{GSH}^{*}$ and $\mathrm{hmGSH} \mathrm{H}^{* *}$ (the chief enzymes which help the plant cope up) synthesis during heat stress in wheat. There is yet another group of genes known as the WRKY genes which help in regulation of both of the above stresses. Hence, we have focused our attention to the work done in the conversion of winter wheat occurring at the time of green revolution into summer wheat. Modifications in the above gene groups of the present day wheat can lead to the origin of a new variety of wheat which could be tolerant to both of the extremes of stresses and could be grown throughout the year with minimum attention.
\end{abstract}

*GSH - Reduced glutathione, ** hmGSH - Reduced hydroxymethyl glutathione

Keywords: GSH, hmGSH, VRN1, VRN2, VRN3, COR genes, WCS120, HSP17, HSP70, DREB, WRKY.

\section{Introduction}

Wheat is the member of the Triticeae group of cereals and indisputably one of the major food crops of the world and a foundation of human nutrition worldwide. In addition to its basic caloric value, wheat has its high protein content too. Traditionally, genetic improvement in wheat is generated by using extensive crossing program and then systematically selection of useful new combinations [McIntosh, 1998, Bedo' et al, 1998, Sahrawat et al, 1998]. Wheat is one of the most important staple food crops of the world, occupying 17\% (one sixth) of crop acreage worldwide, feeding about $40 \%$ (nearly half) of the world population and providing $20 \%$ (one fifth) of total food calories and protein in human nutrition [IUFoST Scientific Information Bulletin, Feb 2005]. The world's poor will need to rely more heavily on biotechnology-related improvements in crop and animal yields and use of marginal lands if the UN's World Food Summit goals of reducing the world's poverty to $50 \%$ of 1996 levels by 2015 are to be attained. The needs will not be the same in future but will increase as the population increases. This will include more of food production, environmental protection, reducing postharvest losses and increasing food quality, both sensory and nutritional. The predicted rise in the population of the world will result in the need for twice or three times the amount of food currently available.

Growth rates of yields have slowed during the period between 1987 and 2001[Huang et al, 2002, Rosegrant et al, 1999] .Moreover, the world's population will reach 8 billion by 2025 and it has been estimated that food and feed production must continue to rise annually by $1.2 \%$ to satisfy the demand of the world's population. Past success, therefore, does not guarantee a food abundant world in coming decades. Hence the genetic improvement of wheat has received considerable attention worldwide over the years with the purpose of increasing the grain yield.

Abiotic stresses such as drought, salinity or low and high temperature are major environmental factors that have adverse effects on plant growth and limit crop production by inducing oxidative stress during which reactive oxygen species accumulate [Valliyodan, 2006]. They also trigger a wide range of plant responses, including changes in various biochemical and physiological processes and changes in gene expression thereby acquiring stress tolerance [Shinozaki et al, 2003, Xiong et al, 2002, Zhu, 2002]. Plant responses to stresses at transcription level have been extensively studied, which has established that changes in gene expression result from a complex signal transduction cascade that commences with perception and subsequent transduction of 
stress signals, and then leading to the activation of various stress-responsive gene expression [Seki, 2003, Shinozaki, 2003, Zhu, 2001] .So there is a need to produce such a variety of wheat(it being a staple crop, if such crops perish, can cause potential harm to the economy and health of the nation) that is able to cope up with pre advent of seasonal conditions as seen oflate.

\section{Techniques For Genetic Transformation Of Wheat \\ 2.1 Tissue Culture and Regeneration: A Prerequisite for Genetic Transformation of Wheat}

Most methods of plants transformation applied to GM crops require that a plant is regenerated from isolated plant cells or tissues which have been genetically transformed. This regeneration is conducted in-vitro so that the environment and growth medium can be manipulated to ensure a high frequency of regeneration.

\subsection{Genetic Transformation of Wheat Employing Microprojectile Bombardment}

High velocity microprojectiles are used to deliver nucleic acids into living cells by a technique known as microprojectile bombardment or biolistic transformation. To transform plants biolistically, DNA is coated onto suitable metal particles, usually gold or tungsten, which are chemically inert. These are blasted into plant tissue using a biolistic device driven by a gas, usually helium. A series of baffles or mesh screens is used to reduce cell death. The type of plant tissue used can vary between different plant species, the most common being immature embryos, embryo genic callus and meristems.

\subsection{Genetic Transformation of Wheat Mediated By Agrobacterium Tumefaciens}

The Ti plasmid, found in cells of the bacterium known as Agrobacterium tumefaciens, can be used to shuttle exogenous genes into host plant cells. This type of gene transfer requires two steps: 1) the endogenous, tumor-causing genes of the T DNA must be inactivated and, 2) foreign genes must be inserted into the same region of the Ti plasmid. The resulting recombinant plasmid, carrying up to approximately 40,000 base pairs of inserted DNA and including the appropriate plant regulatory sequences, can then be placed back into the A. tumefaciens cell. That cell can be introduced into plant cell protoplasts either by the process of infection or by direct insertion.

Once in the protoplast, the foreign DNA, consisting of both T DNA and the inserted gene, incorporates into the host plant genome. The engineered protoplast - containing the recombinant T DNA - regenerates into a whole plant, each cell of which contains the inserted gene. Once a plant incorporates the T DNA with its inserted gene, it passes it on to future generations of the plant with a normal pattern of Mendelian inheritance.

\subsection{Reporter and Selectable Marker Genes for Wheat Transformation}

Initial steps for genetic transformation involve delivery of a gene cassette into recipient cells followed by analysis of the expression of delivered gene. The results of the above events can be detected by assaying the expression of a reporter gene introduced into plant cell cultures or intact tissues. The reporter genes produce a visible effect, directly or indirectly, due to their activity in the transformed cells. Analysis of reporter gene expression does not require the integration of the transgene into the host genome and is commonly used to test promoter and gene functions. Initial studies on the introduction of foreign DNA to wheat have relied on the use of the $E$. coli gene for the enzyme chloramphenicol acetyl transferase (cat). GUS enzyme hydrolyzes b glucuronide compounds and gives reaction products that can be quantified spectrophotometric or spectrofluorometrically. The gus reporter gene system is extremely useful for optimization of parameters for genetic transformation, due to the availability of a simple histochemical detection procedure. One of the major limitations of gus reporter gene system, however, is the destructive nature of its assay.

The varied frequency of DNA delivery in cells of different explants has necessitated the development of methods for efficient selection of cells that carry and express the introduced gene sequences. The selection regimes for transformed cells are based of the expression of a gene termed as the selectable marker producing an enzyme that confers resistance to a cytotoxic substance, often an antibiotic or a herbicide. The most commonly used selection marker in wheat transformation is the bar gene (bialaphos resistance gene) encoding for phosphinothricin acetyl transferase (pat). Both bar and pat genes isolated from different Streptomyces species, encode for phosphinothricin acetyl transferase. Amongst the antibiotic resistance markers, the bacterial neomycin phosphotransferase II (nptII) gene providing resistance to aminoglycoside antibiotics is commonly used in wheat transformation. Herbicide resistance genes offer an alternative to antibiotic-resistant markers. Genes coding for 5-enolpyruvyl-shikimate-3-phosphate synthase (EPSPS), a critical enzyme for aromatic amino acid biosynthesis, and phosphinothricin acetyl transferase (PAT) provide tolerance to glyphosate and glufosinate ammonium herbicides, respectively. The enolpyruvylshikimate-phosphate synthase (CP4) gene isolated from Agrobacterium strain CP4 and the glyphosate oxidoreductase $(G O X)$ also provide tolerance to glyphosate by degrading glyphosate into aminomethyl phosphoric acid, and have been added to the list of selectable markers available for wheat. Recently mannose-6-phosphate isomerase (MPI), encoded by manA from E. coli, has been developed as a selectable marker for wheat. This marker allows transformants to grow on mannose as a sole 

carbon source and has a positive mode of action which encourages the growth of the transformed tissues rather than just permitting it.

Table 1

Some examples on the production of abiotic stress tolerant transgenic plants

\begin{tabular}{|c|c|c|c|c|c|}
\hline No. & Gene & Protein & Source & Role in Cell & Transhost \\
\hline 1. & Gpat & $\begin{array}{l}\text { Glycerol 3-phosphate } \\
\text { acyltransferase }\end{array}$ & $\begin{array}{l}\text { Cucurbita maxima, } \\
\text { A. thaliana }\end{array}$ & Fatty acid unsaturation & N. tabacum \\
\hline 2. & Sod & Superoxide dismutase & N. plumbaginifolia & Superoxide dismutation & Medicago sativa \\
\hline 3. & tps 1 & Trehalose 6-phosphate synthase & A. thaliana & Trehalose biosynthesis & N. tabacum \\
\hline 4. & $\operatorname{cod} A$ & Choline oxidase & Arthrobacter globiformis & $\begin{array}{l}\text { Glycine-betaine } \\
\text { biosynthesis }\end{array}$ & A. thaliana \\
\hline 5. & $a f p$ & Anti-freeze protein & Synthetic & $\begin{array}{l}\text { Inhibits growth of ice and } \\
\text { recrystallization }\end{array}$ & S. tuberosum \\
\hline 6. & hva 1 & LEA protein & Hordeum vulgare & Unknown & O. sativa \\
\hline 7. & $\operatorname{bet} A$ & Choline dehydrogenase & E. coli & $\begin{array}{l}\text { Glycine-betaine } \\
\text { biosynthesis }\end{array}$ & N. tabacum \\
\hline 8. & $\operatorname{sac} B$ & Levan sucrose & A. subtilis & Fructan biosynthesis & N. tabacum \\
\hline
\end{tabular}

\section{Genes Contributing Towards Coping With Environmental Stress}

Mutations in the VRN1 promoter region [Yan et al, 2004, Yan et al, 2003, Yan et al, 2004] or in the first intron [Fu et al, 2005, vonZitzewitz et al, 2005] are completely linked to a dominant spring growth habit in barley and wheat. In hexaploid wheat, a dominant VRN1 allele at any one of the three genomes is sufficient to confer a spring growth habit [Cockram et al, 2007].

VRN1 has been mapped on the long arm of homologous group 5 [Shitsukawa et al, 2007, Law et al, 1976, Dubcovsky et al, 1998], in the same chromosome region associated with quantitative trait loci (QTL) for frost tolerance. It is still unresolved whether this frost tolerance locus, designated as FR1, is just a pleiotropic effect of VRN1 [Limin et al, 2002, Limin et al, 2006] or a separate gene [Laurie et al, 1995, Galiba et al, 1995, Hayes et al, 1993].

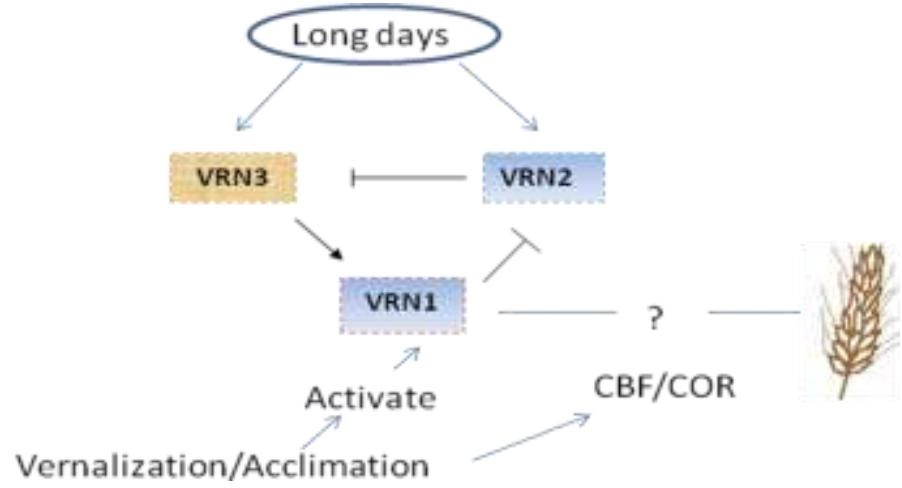

FIGURE 3.1 shows a diagrammatic representation of how vernalization/acclimation controls VRN and CBF complexes.

In populations segregating for the vernalization gene VRN2, FR1 is not detected, and a significant effect on frost tolerance is detected at the VRN2 locus. This suggests that differences in vernalization requirement are sufficient to determine significant differences in frost tolerance. Deletions of VRN2 result in recessive spring growth habit.

Among the cold-regulated genes, COR14b and WCS120 are well characterized in wheat and barley. These genes are differentially expressed in freezing-sensitive and freezing-tolerant plants under both laboratory and field conditions. The COR14b gene encodes a polypeptide that accumulates in the stromal fraction of the chloroplasts in the presence of light, and its transcription is specifically induced by low temperature [Crosatti et al, 1995]. The COR14b protein helps to protect the photosynthetic apparatus from photodamage during light exposure following freezing [Rapacz et al, 2008]. WCS120 (=DHN5) belongs to the dehydrin group of proteins and is likely involved in low temperature-induced physiological dehydration. During cold acclimation, the accumulation of the WCS120 protein shows a positive correlation with freezing tolerance [Sarhan et al, 1997]. Increased transcript levels of COR14b and WCS120 are observed in most winter cereals when plants are exposed to temperatures between 2 and $48 \mathrm{C}$. However, when plants are exposed to milder cold temperatures (e.g. 12-15 8C) the induction profiles of these two genes show intraspecific variation. Crosses between 
accessions that differed in their COR14b and WCS120 transcription levels at 12-15 8C were used to map these expressions QTL in T. monococcum [Stockinger et al, 1997, Va'gu' jfalvi et al , 2003, Crosatti et al , 1995, Rapacz et al , 2008, Sarhan et al 1997, Knox et al, 2008] and barley [Sutka, Snape, 1989]. In both species the peak of the expression QTL for COR14b and WCS120 mapped to the long arm of chromosome 5, approximately $30 \mathrm{cM}$ proximal to VRN1. This chromosome region differs from the chromosome location of the COR14b and WCS120 genes themselves, indicating that this is a trans-acting expression QTL. The expression QTL for COR14b overlaps with a QTL for survival to freezing temperatures in both diploid wheat [Stockinger et al, 1997] and barley [Sutka, Snape, 1989]. The locus under the peaks of these overlapping QTL was designated FR2 (Frost Resistance 2) [Stockinger et al, 1997], and was shown to play also an important role in frost tolerance in polyploid wheat [Ba'ga, 2007, Va'gu' jfalvi, 2005]. A high-density map of the FR2 region in diploid wheat confirmed that the peaks of the QTL for frost tolerance and for COR14b and WCS120 expression both map within a small $0.7 \mathrm{cM}$ region [Sarhan et al, 1997]. This suggests that these two traits are either pleiotropic effects of the same gene or the result of tightly linked genes within this small genetic interval.

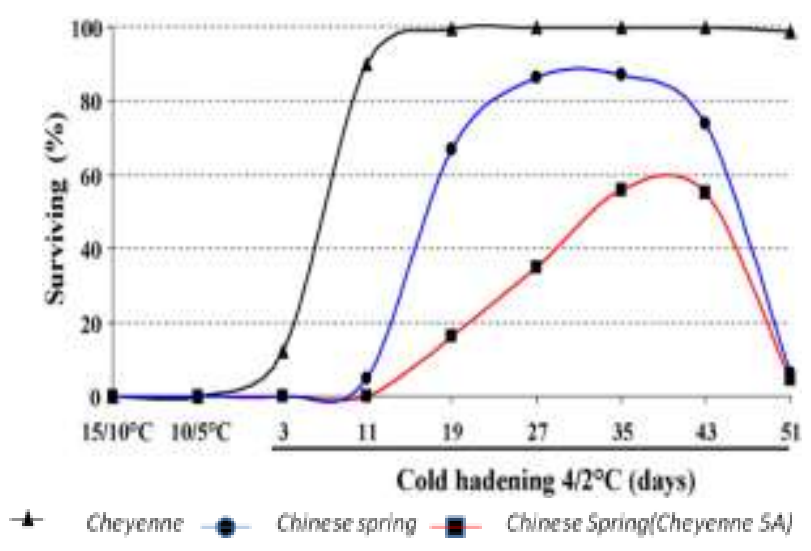

FIGURE 3.2

Effect of cold hardening duration on wheat frost tolerance. Frost tolerant cheyenne and chinese spring(Cheyenne 5A) chromosome substitution line and frost sensitive chinese spring plants were cold hardened for different number of days and subjected to frost tests (_10 $8 C$ ). Frost tolerance was evaluated as percent survival. Plants were initially grown at 15/10 8C (day/night), then pre-hardened at 10/5 8C and finally exposed to cold hardening at 4/2 8C for 3,11,19,27, 35, 43 and 51 days before the frost test

The CBF genes, also known as Dehydration Responsive Elements (DRE)-binding factors, bind to the conserved core sequence CCGAC [C-repeat (CRT)/dehydration element (DRE)] in the promoters of many dehydration-responsive genes including those with early response to dehydration and cold [Stockinger et al, 1997, Liu et al, 1998]. Transcription profiling and high-density mapping strategies have been used to identify possible candidate genes for frost tolerance within the FR2-CBF cluster.

The CBF genes have been identified in T. monococcum using a high-density mapping approach. Using lines with recombination events within the FR2 locus, the main differences in frost tolerance and in the differential regulation of COR14b and WCS120 genes were mapped to the central region of the CBF gene cluster, completely linked to TmCBF12, TmCBF14 and TmCBF15 [Sarhan et al, 1997]. A smaller effect on frost tolerance was also associated with TmCBF16. This set of CBF genes partially overlaps with those described above in polyploid wheat [Galiba et al, 1995]. The cold acclimation process in plants is genetically programmed and is associated with an increased freezing tolerance (FT) resulting from the expression of many genes [Stockinger et al, 2007].

The cDNA of TaSrg6 (Accession no. EF063153) was amplified by PCR using ds-cDNA from PEGtreated roots. Sequence analysis indicated that TaSrg6 was of $1500 \mathrm{bp}$ in length with an open reading frame of 1098 bp. The expression patterns of TaSrg6 gene were determined by real-time quantitative PCR analysis under different stress conditions, including PEG, high salt, low temperature and abscisic acid (ABA) treatment. TaSrg6 was expressed at very low level in the root tissue under normal growth condition, and its expression level did not change in the non-stressed treatment over different time courses [Tong et al, 2007].

Experimental studies have indicated the contribution of chromosome 5a of wheat involved in the regulation of heat-induced changes in both the hmGSH and GSH contents since the alterations in the amount of these thiols in the 5A chromosome substitution lines resembled to those observed in the donor genotypes of this chromosome. The difference in heat tolerance in different wheat genotypes may be associated with multiple processes and mechanisms involving HSPs, transcription factors, and other stress related genes.. Heat acclimation has little effects on gene expression under prolonged treatments but affects gene expression in wheat under short-term heat stress.

Quantitative differences were observed in the synthesis of HSP 22 and more subtle differences detected in the synthesis of major HSP 16 and 17. Increased synthesis of HSP 33, 42, and 62 was observed in the heat 
tolerant Mustang. Several high molecular wt $\operatorname{HSP}(83,85,92,94 \mathrm{kD})$ were synthesized at elevated levels in the heat susceptible variety.

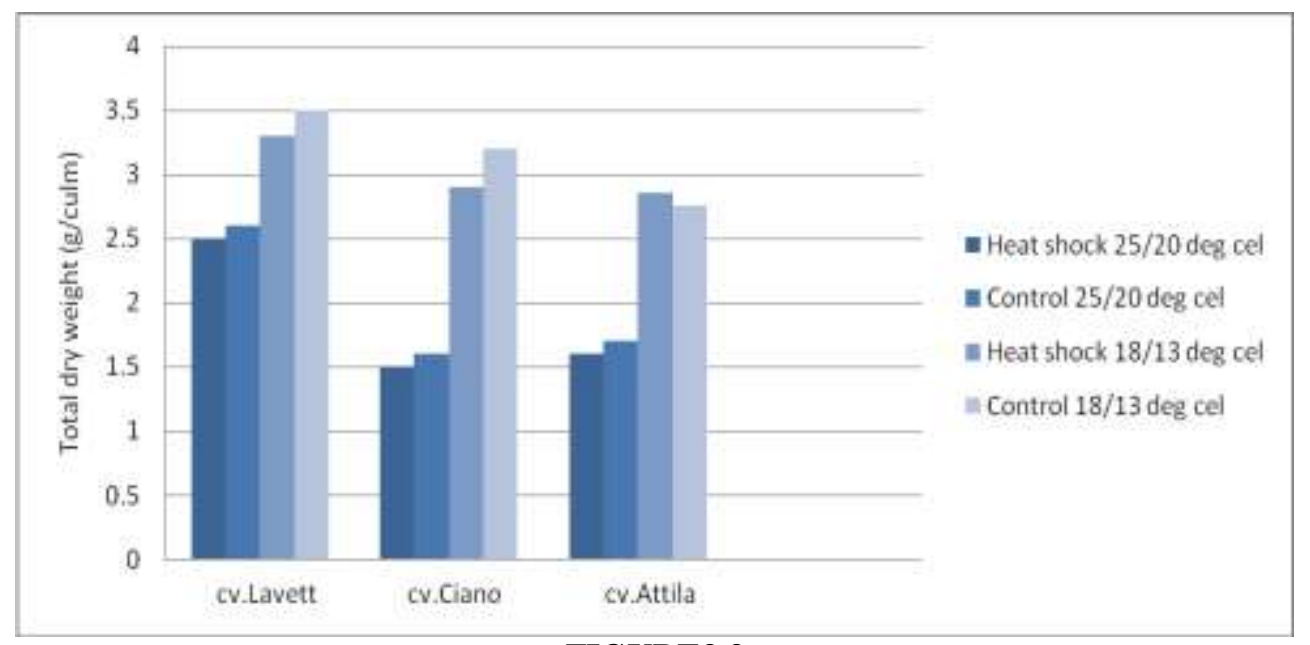

FIGURE3.3

Effects of two growth temperatures, $18 / 13$ or $25 / 20^{\circ} \mathrm{C}$, and a heat shock at early grain filling on total dry weight at the final harvest for three wheat cultivars.

WRKY proteins are involved in various physiological processes, including biotic and abiotic stress responses, hormone responses and development. However, no systematic identification, expression and function analysis of $W R K Y$ genes in wheat were reported. In this study, we isolated 15 wheat cDNAs with complete open reading frame (ORF) encoding putative WRKY proteins using in silico cloning. Phylogenetic analysis indicated that the 15 wheat $W R K Y$ genes belonged to three major WRKY groups. Expression analysis revealed that most genes expressed drastically in leaf, except TaWRKY10 which expressed in crown intensively. Four genes were strongly up-regulated with the senescence of leaves. Eight genes were responsive to low temperature, high temperature, $\mathrm{NaCl}$ or PEG treatment. Moreover, differential expression patterns were also observed between wheat hybrid and its parents, and some genes were more responsive to PEG treatment in the hybrid. These results demonstrated that wheat $W R K Y$ genes are involved in leaf senescing and abiotic stresses. And the changed expression of these $W R K Y$ genes in hybrid might contribute to the heterosis by improving the stress tolerance in hybrids [Wu et al, 2008].

Table 2:

Characteristic features of plant stress proteins

\begin{tabular}{ll}
\hline \multicolumn{1}{c}{ Stress Protein } & \multicolumn{1}{c}{ Induction Agent } \\
\hline HSPs (heat shock proteins) & $\begin{array}{l}\text { Mostly by high temperature stress; } \\
\text { also by water stress, salt stress, in } \\
\text { some cases, also by abscisic acid }\end{array}$
\end{tabular}

Osmotic stress proteins such as WSPs (water stress proteins) and SSPs (salt stress proteins)

ANPs (Anaerobic proteins)

Cold stress proteins such as COR (cold-regulated proteins), KIN, LIP, BLT, WCS, etc.
Mostly by low water availability and salt stress, also induced by $\mathrm{ABA}$

Mostly by anaerobic stress (cause by flooding or submergence stress)

Mostly by low temperature stress; also by osmotic, oxidative stress and $\mathrm{ABA}$

\section{Classified as low molecular weight HSPs and high molecular weight HSPs, highly conserved amino acid sequence, nucleotide sequence of the corresponding genes is also considered, stress-inducibility of hs genes governed by HSEs (heat shock elements), selected HSPs are shown to act as chaperones}

Varied molecular weights and cellular locations, these proteins are mostly the enzymes involved in diverse functions such as production of different osmolytes, protein degradation, signal transduction events, gene regulation and transport. Roles of some WSPs is not well defined (i.e. such as for dehydrins, Late embryogenesis, Abundant proteins etc.)

Discovered initially in maize and later shown to be universally present, most of the ANPs have been shown to be the enzymes of the fermentative or the glycolytic pathway, most genes encoding ANPs contain AREs (Anaerobic Response Elements) in their promoters

Conserved proteins of varied sizes and functions, most COR genes contain specific nucleotide sequence that stimulate transcription in response to low temperature

The molecular mechanism by which aluminium inhibits cell division in plant roots under acidic conditions is not understood. We have used Northern hybridizations to monitor changes in gene expression following Al treatment of two wheat cultivars: Warigal, an Al-sensitive variety, and Waalt, an AAl-tolerant 
variety. Steady state transcript levels of two heat shock genes, hsp17 and hsp70, were not increased in roots by the $\mathrm{Al}$ treatment. Levels of histone $\mathrm{H} 3$ and $\mathrm{H} 4$ transcripts were reduced by long-term exposure to inhibitory $\mathrm{Al}$ treatments in both wheat cultivars, consistent with previous reports that histone genes are expressed primarily in dividing cells. In addition, we have isolated a wheat gene for $S$-adenosyl-l-homocysteine hydrolase (SHH), and demonstrated that its mRNA is downregulated by long-term exposure to inhibitory levels of Al [Richards, Gardner, 1994].

\section{Utilizing The Above Simultaneously}

The above mentioned techniques provide us a boon and open scope for the creation of so proposed "Evergreen Wheat". The identified genes for the various types of stresses that the plant has to encounter provide us the target locations. It is not that the above mentioned genes are absent in all of the wheat varieties. The genes may or may not be present and even if all of them are present, they might not be having the signals for them to be expressed completely. Experiments have been conducted to improve heat, frost, drought stress resistance in the crop. Only if all of the efforts are put together such that the concerned genes are all activated in a single variety can the dream of Evergreen wheat can come true.

This may be not only a high technology process but even crossing and selfing must be involved for the generation of pure lines so that no other gene hampers the functioning of the concerned genes. If such a condition arises then gene silencing should be adopted as a measure. It is possible that the initially produced wheat may be deficient in some other gene but those can be dealt with by crossing with the variety possessing hybrid vigor for that particular trait. The techniques can employ placing of transcription initiator sequences before the gene of interest begins or even via plasmid transfer, sequences for the synthesis of signals for the activation of these genes can be inserted into the wheat genome so that the plant becomes self dependent. On encountering stress, the signal proteins will be synthesized and the genes for coping up with the stress will be activated so long as the stress factor is present in the environment. This may also require abrupt termination of transcription and translation as per the requirement.

\section{Safety}

Advances in scientific fields such as toxicology and immunology put science in a better position to evaluate and recognize potential hazards and assure product safety of any new foods derived from biotechnology. As with any food or food process, safety remains paramount. It is seriously considered before any product is placed into the food production or distribution chain [Bradford, 1976].

\section{Conclusion}

Significant progress during the last two decades has been made in different areas of wheat genomics research. These include development of thousands of molecular markers (including RFLPs, SSRs, AFLPs, SNPs, and DArT markers), construction of molecular genetic and physical maps (including radiation hybrid maps for some chromosomes) with reasonably high density of markers, development of more than 1 million ESTs and their use for developing functional markers, and the development of BAC/BIBAC resources for individual chromosomes and entire sub genomes to facilitate genome sequencing. Functional genomics approaches like TILLING, RNAi, and epigenetics have also been utilized successfully, and a number of genes/QTL have been cloned to be used in future wheat improvement programs. Organellar genomes including chloroplast and mitochondrial genomes have been fully sequenced, and we are at the threshold of initiating a major program of sequencing the gene space of the whole nuclear genome in this major cereal. The available molecular tools also facilitated a revisit of the wheat community to the problem of origin and evolution of the wheat genome and helped QTL analysis (including studies involving LD and association mapping) for identification of markers associated with all major economic traits leading to the development of major markeraided selection (MAS) programs for wheat improvement in several countries.

\section{Acknowledgements}

We are grateful to our parents for the efforts and the co-operation they provided. We thank our friends and teachers for the screening they provided.

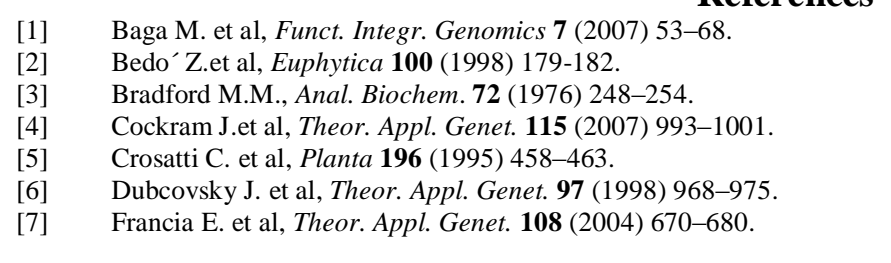


[8] Fu D. et al, Mol. Genet. Genomics 273 (2005) 54-65.

[9] Galiba G. et al, Theor. Appl. Genet. 90 (1995) 1174-1179.

[10] Hayes P.M.et al, Genome 36 (1993) 66-71

[11] Huang J. et al, Nature 418 (2002) 678-684.

[12] Jansen R.C., Nap J.P., Trends Genet. 17 (2001) 388-391.

[13] Knox A.K. et al, Plant Mol. Biol. 67 (2008) 257-270.

[14] Laurie D.A. et al, Genome 38 (1995) 575-585.

[15] Law C.N.et al., Heredity 36 (1976) 49-58.

[16] Limin A.E., Fowler D.B.. Ann. Bot. 89 (2002) 579-585.

[17] Limin A.E., Fowler D.B., Planta 224 (2006) 360-366.

[18] Liu Q. et al, Plant Cell 10 (1998) 1391-1406.

[19] McIntosh R.A., Euphytica 100 (1998) 19-34.

[20] Rapacz M. et al, Ann. Bot. 101 (2008) 689-699.

[21] Richards K.D., Gardner R.C. Plant Sci. 98 (1994) 37-45.

[22] Rosegrant M.W. et al, Proc. Nutr. Soc. 58 (1999) 219-234.

[23] Sahrawat A. K. et al, Plant Sci. 165 (2003) 1147-1168.

[24] Sarhan F. et al, Physiol. Plantarum 101 (1997) 439-445.

[25] Seki M. et al, Curr. Opin. Biotechnol. 14 (2003) 194-199.

[26] Shinozaki K.et al, Curr. Opin. Plant Biol. 6 (2003) 410-417.

[27] Shinozaki K., Dennis E.S., Curr. Opin. Plant Biol. 6 (2003) 405-409.

[28] Shitsukawa N.et al, Genes Genet. Syst. 82 (2007) 167-170.

[29] Stelmakh A.F., Euphytica 36 (1987) 513-519.

[30] Stockinger E.J. et al, Plant J. 51 (2007) 308-321.

[31] Stockinger E.J. et al, Proc. Natl. Acad. Sci. U.S.A. 94 (1997) 1035-1040.

[32] Sutka J. et al, Theor. Appl. Genet. 99 (1999) 199-202.

[33] Sutka J., Snape J.W., Euphytica 42 (1989) 41-44.

[34] To' th B. et al, Theor. Appl. Genet. 107 (2003) 509-514.

[35] Tong S. et al, Plant Sci. 172 (2007) 1079-1086.

[36] Va'gu' jfalvi A. et al, Mol. Genet. Genomics 269 (2003) 60-67.

[37] Va'gu' jalvi A. et al, Mol. Genet. Genomics 274 (2005) 506-514.

[38] Valliyodan B., Nguyen H.T., Curr. Opin. Plant Biol. 9 (2006) 189-195.

[39] VonZitzewitz J. et al, Plant Mol. Biol. 59 (2005) 449-467.

[40] Wu H. et al, Nat. Sci. 18 (2008) 697-705

[41] Xiong L. et al, Plant Cell. 14 (2002) S165-S183.

[42] Yan L.et al, Science 303 (2004) 1640-1644.

[43] Yan L. et al, Proc. Natl. Acad. Sci. U.S.A. 100 (2003) 6263-6268.

[44] Yan L. et al, Theor. Appl. Genet. 109 (2004) 1677-1686.

[45] Zhu J.K., Annu. Rev. Plant Biol. 53 (2002) 247-273.

[46] Zhu J.K., Curr. Opin. Plant Biol. 4 (2001) 401-406. 